J. Austral. Math. Soc. 20 (Series A) (1975), 1-17.

\title{
AN AXIALLY SYMMETRIC FORCED CONVECTION PROBLEM
}

\author{
J. A. BELWARD
}

(Received 4 April 1972; revised 18 June 1972)

Communicated by A. F. Pillow

\begin{abstract}
A simple diffusion-convection heat transfer model is formulated which leads to an axially symmetric partial differential equation. The equation is shown to be closely related to a second one which is adjoint to the original equation in one variable can and be interpreted as a description of another diffusion-convection model. Fundamental solutions of the original equation are constructed and interpreted with reference to both models. Some boundary value problems are solved in series form and integral representations of the solutions are also given. The boundary value problems are shown to be equivalent to an integral equation and the correspondence between the two formulations is understood in terms of the two diffusion-convection problems. A Péclet number is defined in one of the boundary value problems and the behaviour of the solutions is studied for large and small values of this parameter.
\end{abstract}

\section{Introduction}

Axially symmetric partial differential equations have attracted the interest of many researchers and a considerable quantity of information about these equations has been accumulated in the literature (see Weinstein (1953), Burns (1967), and Colton (1969), for example). The results obtained to date have been mainly theoretical in character and the purpose of this paper is to describe some more practical results. These have been obtained with the aid of a simple heat transfer model which gives rise to an axially symmetric boundary value problem. In this problem the temperature is governed by the equation.

$$
\frac{\partial^{2} T}{\partial z^{2}}+\frac{\partial^{2} T}{\partial \rho^{2}}+\frac{1-\alpha}{\rho} \frac{\partial T}{\partial \rho}-2 \lambda \frac{\partial T}{\partial z}=F(r),
$$

where $\lambda$ and $\alpha$ are real constants, $F(r)$ is a singular functional concentrated on an arc or a point and $(z, \rho, \phi)$ are the coordinates on a point in a cylindrical polar coordinate system. Equation (1.1) is the main focus of interest in the paper.

Fundamental solutions of equation (1.1) will be constructed and their relevance to the properties of the physical model will be discussed. Some boundary value problems involving conditions prescribed on the axis of symmetry will also be solved.

(C) Copyright Australian Mathematical Society 1975

Copyright. Apart from any fair dealing for scholarly purposes as permitted under the Copyright Act, no part of this JOURNAL may be reproduced by any process without written permission from the Treasurer of the Australian Mathematical Society. 
The latter problems lead naturally to the integral equation

$$
\begin{array}{r}
\frac{2^{\alpha / 2-3 / 2} \lambda^{1-\alpha}}{\pi^{3 / 2} \Gamma(1-\alpha / 2)} \int_{c}^{d} g(t) e^{\lambda(z-t)}(\lambda|z-t|)^{(\alpha-1) / 2} K_{(\alpha-1) / 2}(\lambda|z-t|) d t=f(z), \\
c<z<d,
\end{array}
$$

where $\alpha$ and $\lambda$ are the same constants as those appearing in equation (1.1) and $K_{v}(w)$ is the modified Bessel function which is the solution of the equation

$$
w^{2} y^{\prime \prime}+w y^{\prime}-\left(w^{2}+v^{2}\right) y=0
$$

with the asymptotic form $K_{t}(w) \sim(\pi / 2 w)^{\frac{1}{2}} e^{-w}$ as $w \rightarrow \infty$. The Bessel function has a singularity at the origin which gives the whole kernel a singularity $|z-t|^{\alpha-1}$.

The boundary value problems can be formulated in terms of the partial differential equation (1.1) or in terms of the integral equation (1.2). By interpreting a certain partial differential equation, which is the adjoint of equation (1.1) in $\rho$, as a conservation equation, it is shown that the solutions of these equations can be deduced each from the other (the solution of equation (1.2) is given in another paper by Belward (to appear); the results here are largely restricted to establishing the abovementioned relationship). The behaviour of the solutions of the pronlems for large and small values of $\lambda$ is investigated by approximating the integral equation (1.2).

The physical model which is used to assist and interpret the analysis is a forced convection heat transfer model. The transport mechanisms are those of convection in a prescribed velocity field and of diffusion and the transporting medium is an ideal inviscid fluid. The density, conductivity and specific heat of the fluid are assumed to be unaffected by the temperature and are constant in space and time.

In non-dimensional form the basic equations are

$$
Q=-\nabla T+q T,
$$

which states that the heat flux is the sum of the diffusive and convective fluxes,

$$
q=2 \lambda \hat{z}+\frac{\alpha}{\rho} \hat{\rho},
$$

which describes the velocity field, and

$$
\operatorname{div} Q=F_{1}(r)
$$

which is the heat conservation equation.

The convection field consists of a uniform stream of velocity $2 \lambda$ parallel to the $z$ axis and the velocity due to a mass source of strengh $2 \pi \alpha$ per unit length distributed uniformly along the $z$ axis. The constant $\lambda$ is the Péclet number. If there is a characteristic length in the problem then it will be assumed that this 
length has been scaled to unity. Since the thermal diffusivity has also been taken to be unity, the constant $\lambda$ is genuinely the Péclet number, $U L \kappa^{-1}$, which characterises the ratio of typical magnitudes of the convective flux (by the uniform stream) and the diffusive flux. When equations (1.3), (1.4) and (1.5) are combined, equation (1.1) is obtained with

$$
F(\boldsymbol{r})=\frac{\alpha}{\rho} T(z, \rho) \delta(\rho)-F_{1}(\boldsymbol{r}) .
$$

The former of these terms is due to the mass source, which also acts as a heat source because the fluid is assumed to appear or disappear at the $z$ axis with the ambient temperature of the fluid.

Equation (1.1) can be regarded as an equation which governs the outer solutions of several heat transfer and viscous flow problems. In the temperature problems, the velocity component due to the mass source may be prescribed in the full problem, or it may arise due to the effect of a strong heat source which creates a heat plume and causes suction into the buoyancy jet.

Since we will mostly consider problems in which the boundary conditions are prescribed on some segment of the axis of symmetry, the results will be relevant to problems involving finite bodies, or semi-infinite bodies whose dimensions in the $z$ direction are large compared to their other dimensions (e.g. a paraboloid of revolution).

The rotationally symmetric motion of a viscous fluid can be described (see Pillow (1970)) in equations of the form of equation (1.1), thus:

$$
\begin{array}{r}
\left.\left.\operatorname{div}(-\eta \nabla T+) u+\frac{2 \eta}{\rho} \hat{\boldsymbol{\rho}}\right) T\right)=0 \\
\operatorname{div}\left(-\eta \nabla l+\left(\boldsymbol{u}-\frac{2 \eta}{\rho} \hat{\boldsymbol{\rho}}\right) l\right)=\frac{\partial}{\partial z}\left(\rho^{-4} T^{2}\right)-4 \pi \eta l \delta(\rho),
\end{array}
$$

and

$$
\begin{gathered}
\operatorname{div}\left(-\nabla \psi+\frac{2}{\rho} \hat{\boldsymbol{\rho}} \psi\right)=\rho^{z} l, \\
\boldsymbol{u}=u_{z} \hat{z}+u_{\rho} \hat{\boldsymbol{\rho}}+u \hat{\phi}_{\phi}, \\
u_{z}=\frac{1}{\rho} \frac{\partial \psi}{\partial \rho}, \quad u_{\rho}=-\frac{1}{\rho} \frac{\partial \psi}{\partial z}, \quad u_{\phi}=\frac{T}{\rho}, \\
\rho l=\boldsymbol{\omega} \cdot \hat{\boldsymbol{\phi}} \text { with } \boldsymbol{\omega}=\operatorname{curl} \boldsymbol{u} .
\end{gathered}
$$

$\psi$ is the Stokes stream function, set to zero on the $z$ axis, $l$ is called ring circulation volume density and $\eta$ is the kinematic viscosity. Equations (1.7) and (1.8) are conservation equations for angular momentum and ring circulation respectively, equation (1.9) is a consequence of the definition of the vorticity. 
For flows past finite bodies in an unbounded fluid with free stream velocity $2 \lambda$ at infinity we may use the Oseen approximation and substitute $u=2 \lambda \hat{z}$ in the terms $u l$ and $u T$ of equations (1.8) and (1.7). Then equations (1.7), (1.8) and (1.9) all become special cases of equation (1.1). The form of equations (1.8) and (1.9) shows that these purely viscous problems can also be modelled as diffusion convection problems in which angular momentum and ring circulation are conserved quantities. Finally note that we can also put $\boldsymbol{u}=2 \lambda \hat{z}+(\alpha / \rho) \hat{\boldsymbol{\rho}}$ into these equations. This would be done if the heat were regarded as being transported in a vicsous fluid, and not an inviscid fluid as stated earlier.

Equation (1.2) arises in an elastic half space problem where the medium is isotropic and the Youngs modulus, $E$, varies as a power of the depth, $E=E_{0} d^{(\alpha-1) / 2}$ $1<\alpha<2$. The equation is the Fourier transform of a two-dimensional integral equation, and $f(z)$ is the transform of the prescribed displacement on the surface of the half space and $g(t)$ is the transform of the unknown normal stress at the surface. For $z<c$, and $z>d, g=0$ and $f$ is unknown. For further details of this problem the reader is referred to Koroniev.

Before deriving explicit solutions some general properties can be noted. Properties of the solutions near $\rho=0$ can be determined with $\lambda=0$. For, near $\rho=0$ either diffusion or convection by the line source will be dominant. In contrast, at large distances the values of $\lambda$ are important since convection by the uniform stream is then the dominant effect.

Secondly, following Weinstein [1953], we note that if $T^{*}=\rho^{-a} T$ then

$$
\frac{\partial^{2} T^{*}}{\partial \rho^{2}}+\frac{\partial^{2} T^{*}}{\partial z^{2}}+\frac{1+\alpha}{\rho} \frac{\partial T^{*}}{\partial \rho}-2 \lambda \frac{\partial T^{*}}{\partial z}=\rho^{-\alpha} F(\boldsymbol{r}) .
$$

This implies that a flux vector and a conservation principle can be associated with $T^{*} . T^{*}$ is called " $\alpha$-temperature" and the associated energy quantity is called " $\alpha$-heat". The convection field is the same except that the radial component has its sign reversed. This property is important because certain fundamental solutions which lack a simple interpretation as heat singularities can be recognized as the fields of multipoles of $\alpha$-heat. Note finally that if $T_{1}(r ; \alpha)$ is a solution of (1.13) and $F(\boldsymbol{r})=0$ then a second solution is given by

$$
T_{2}(r ; \alpha)=\rho^{\alpha} T_{1}(r ;-\alpha) \text {. }
$$

\section{Fundamental solutions of the axially symmetric equation, equation (1.1)}

The solutions of equation (1.1) which will be given in this and the next section can be put into two groups, namely fundamental solutions and solutions of certain boundary value problems. The meanings of these terms is self-evident, we commence with fundamental solutions.

If an exponential factor $e^{\lambda z}$ is first removed from $T$ and the new equation 
written in spherical polar $(r, \theta, \phi)$ coordinates, then the following family of separable solutions can be found:

$$
T_{n}(r, \theta)=e^{\lambda z}\left(1-\mu^{2}\right)^{\alpha / 2} C_{n}^{\left(\frac{1}{2}+\alpha / 2\right)}(\mu) r^{(\alpha-1) / 2} K_{n+(1+\alpha) / 2}(\lambda r), n=0,1,2, \cdots,
$$

Here $\mu=\cos \theta$ and $C_{n}^{(\delta)}(w)$ is a Gegenbauer polynomial (see Erdelyi (1954), Volume II). This sequence of solutions is a sequence of multipole expansions, the multipoles being situated at the origin. For $n=0$ the solution (2.1) is the field of a source of heat, for $n=1$ the solution is a linear combination of a source and a dipole, and so on.

To identify these temperature fields we first consider the case $\lambda=0$. For $n=0$ we have

$$
T=\frac{\sin ^{\alpha} \theta}{r} .
$$

By analogy with established results in potential theory one is tempted to identify this as the field of a point source, because of its $r^{-1}$ dependence. Such an argument must be invalid because of the importance of the term $(1-\alpha) / \rho \cdot \partial T / \partial \rho$ at $\rho=0$, although in fact the conclusion is true. It is proved rigorously by showing that

$$
\left(-\nabla^{2}+q \cdot \nabla\right) \frac{\sin ^{\alpha} \theta}{r}=\text { (const.) } \delta(r),
$$

where $q=(\alpha / \rho) \hat{\boldsymbol{\rho}}$. We form the inner product of the left hand side of (2.3) with a $C^{\infty}$ function of bounded support, $\psi$ say. We can then prove that

$$
\int_{\Omega}\left[\left(-\nabla^{2}+q \cdot \nabla\right) \frac{\sin ^{\alpha} \theta}{r}\right] \psi d v=\frac{4 \pi^{\frac{3}{2}} \Gamma(1+\alpha / 2)}{\Gamma\left(\frac{1}{2}+\alpha / 2\right)} \psi(0) .
$$

This implies that (2.2) is the field of a point source of heat of strength $4 \pi^{\frac{3}{2}} \Gamma(1+\alpha / 2) \Gamma^{-1}\left(\frac{1}{2}+\alpha / 2\right)$

Corresponding to $\lambda=0$, further separable solutions are

$$
T_{n}=\left(1-\mu^{2}\right)^{\alpha / 2} C_{n}^{(1+\alpha / 2)}(\mu) r^{-1-n}, \quad n=1,2, \cdots .
$$

These are the fields of a $2^{n}$-pole of heat aligned along the $z$ axis. To prove this we observe that

$$
\frac{\partial^{p}}{\partial z^{p}}\left(\left(1-\mu^{2}\right)^{\alpha / 2} C_{0}^{\left(\alpha / 2+\frac{1}{2}\right)}(\mu) r^{-1}\right)=(-1)^{p} p !\left(1-\mu^{2}\right)^{\alpha / 2} C_{p}^{\left(\alpha / 2+\frac{1}{2}\right)}(\mu) r^{-p-1},
$$

(a result which can be proved by induction). Then, because equation (1.1) is autonomous in $z$ it follows that

$$
\left(-\nabla^{2}+q \cdot \nabla\right) T_{n}=(\text { const. })(\hat{z} \cdot \nabla)^{n} \delta(\boldsymbol{r}) .
$$


Finally, the identification of the set (2.1) as the elements of a multipole expansion is implied by the expansion of the Bessel function for small values of $r$. To be strictly rigorous in the above statements the condition $\alpha>-2$ must be added. For $\alpha \leqq-2$ the sets (2.1) and (2.5) remain solutions of equation (1.1) but the interpretations given above are no longer applicable.

We note for later use that the field

$$
T=\frac{1}{\Gamma(1+\alpha / 2) \pi^{\frac{3}{2}} 2^{\frac{3}{2}+\alpha / 2}} e^{\lambda z} \sin ^{\alpha} \theta r^{(\alpha-1) / 2} K_{(1+\alpha) / 2}(\lambda r)
$$

is the field of a point source of heat at the origin, of strength unity.

On applying equation (1.14) to the sequence of solutions (2.1) we obtain a second set thus:

$$
T_{m}(r, \theta)=e^{\lambda z} C_{m}^{\left(\frac{1}{2}-\alpha / 2\right)}(\mu) r^{(\alpha-1) / 2} K_{m+(1-\alpha) / 2}(\lambda r), m=0,1,2, \cdots .
$$

These solutions are as equally important as the set (2.1). They are the temperature field of linear combinations of multipoles of $\alpha$-heat. This assertion is proved by forming $T_{m}^{*}(r, \theta)=\rho^{-\alpha} T_{m}(r, \theta)$, thus

$$
T_{m}^{*}(r, \theta)=e^{\lambda z}\left(1-\mu^{2}\right)^{-\alpha / 2} C_{m}^{\left(\frac{1}{2}-\alpha / 2\right)}(\mu) r^{-(\alpha-1) / 2} K_{m+(1+\alpha) / 2}(\lambda r), m=0,1,2, \cdots
$$

Now since the fields (2.1) satisfy equation (2.6) it follows from the remarks leading to equation (1.14) that the set (2.9) satisfies

$$
\left(-\nabla^{2}+q^{*} \cdot \nabla\right) T_{m}^{*}=\text { (const.) }(\hat{z} \cdot \nabla)^{m} \delta(r),
$$

where $q^{*}=-(\alpha / \rho) \hat{\boldsymbol{\rho}}$, thus the fields $T_{m}^{*}$, and thus $T_{m}$, are multipole fields of $\alpha$-heat, as asserted. Note in particular that for $m=0$ the field (2.8) is that of a source of $\alpha$-heat of strength $2^{3 / 2-\alpha / 2} \pi^{3 / 2} \Gamma(1-\alpha / 2)$. Note also that the condition $\alpha<2$ should preface the statements concerning equations $(2.8,9$, and 10$)$ in the same way that $\alpha>-2$ was imposed earlier.

It can be seen that the fields (2.8) are well behaved for all values of $r$ other than $r=0$, whereas the solutions (2.1) are either zero or infinite on the $z$ axis according to the sign of $\alpha$. In the latter case it is the values of $T^{*}$ which are well behaved, and thus we have an interesting duality; multipoles of heat have finite $\alpha$-temperature everywhere, whereas multipoles of $\alpha$-heat have finite temperature everywhere (with the exception of the origin in both cases). This points to the need for a condition on $T$ or $T^{*}$ on the $z$ axis in the complete statement of any well posed boundary value problem involving equation (1.1). This is a consequence of the singular convection field.

The behavior of the temperature on the axis in the presence of a heat (as opposed to $\alpha$-heat) singularity is entirely consistent with the physics of the problem. For, it is well known that if, in the absence of convection, a needle is placed on 
the $z$ axis to release heat into the fluid, then in order to produce a non zero temperature in the fluid the temperature has to be infinite on the needle. When $\alpha$ is positive the radial convection takes heat away from the axis rapidly tending to reduce the temperature there, whereas for $\alpha$ negative the convection field tends to prevent heat escaping from the axis. The fact that the field of a point source of heat has $T \sim \rho^{\alpha}$ as $\rho \rightarrow 0$ is consistent with this behaviour.

The asymptotic behaviour of these functions and the dispersion of the heat (or $\alpha$-heat) in various directions in the fluid is information worthy of interest. The asymptotic expansions can be written down immediately, thus with $z=r \cos \theta$,

$$
T_{n} \sim\left(1-\mu^{2}\right)^{\alpha / 2} C_{n}^{\left(\frac{1}{2}+\alpha / 2\right)}(\mu) e^{-\lambda r(1-\cos \theta)} r^{\alpha / 2-1},
$$

and we obtain a paraboloidal wake which is characteristic of many forced convection problems. There is exponential decay across the paraboloid of revolution $r(1-\cos \theta)=$ a constant, and on the surface of the paraboloid $T \sim r^{-1}$ as $r \rightarrow \infty$ since $\left(1-\mu^{2}\right)^{\alpha / 2} r^{\alpha / 2-1}=[r(1-\cos \theta)]^{\alpha / 2}(1+\cos \theta)^{\alpha / 2} r^{-1}$. The asymptotic form of $(2.8)$ is very similar,

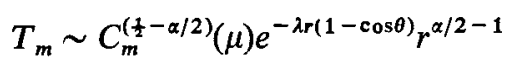

the only difference being that $T \sim r^{\alpha / 2-1}$ on the surface of a paraboloid of revolution now.

With regard to the dispersion of heat within the fluid, in the absence of radial convection it is well known that all heat is carried downstream, and none gets upstream. Exactly the same results hold in the current problems, for any value $\alpha$. Let $M$ equal the heat flux across the plane $z=a$, then

$$
M=\left.\int_{0}^{\infty} Q_{z}\right|_{z=a} 2 \pi \rho d \rho .
$$

It is easily shown, when $T$ is given by equation (3.7), that

$$
M=\left\{\begin{array}{cc}
2^{\alpha / 2+3 / 2} \pi^{3 / 2} \Gamma\left(1+\frac{\alpha}{2}\right), & a>0, \\
0 \quad, & a<0 .
\end{array}\right.
$$

Thus no heat crosses a plane upstream of the source, but it all crosses every plane downstream, so that it must all go downstream. Similar results hold for the distribution of $\alpha$-heat, the calculation is identical to that given above except that $-\alpha$ replaces $\alpha$.

This completes the analysis of solutions in spherical coordinates. We next find some fundamental solutions in cylindrical polar coordinates. It is instructive for us to find the field of a ring source of heat situated at $z=0, \rho=a$, since it helps to understand the behaviour of the solutions of equation (1.1) on the axis of symmetry. The solutions can be found as expansions in terms of the multi- 
pole fields (2.1) or (2.8), but it is preferable to use cylindrical polar coordinates since a closed form solution can then be found.

If a ring source of total strength $M$ is placed at $z=0, \rho=a$, then $T$ must satisfy equation (1.1) with

$$
F(\boldsymbol{r})=-\frac{M}{2 \pi a} \delta(\rho-a) \delta(z) .
$$

One can then solve equation (1.1) by taking Fourier transforms in $z$ and solving the resulting ordinary differential equation in $\rho$. The problem has two admissible solutions which decay at infinity. They are

$$
T=k_{1}(\alpha) e^{\lambda z} \rho^{\alpha} \int_{0}^{\pi} R^{-\alpha / 2-\frac{1}{2}} K_{-\alpha / 2-\frac{1}{2}}(\lambda R) \sin ^{\alpha} t d t,
$$

where

$$
k_{1}(\alpha)=\frac{M \lambda^{\alpha / 2+\frac{1}{\alpha}}}{2^{3 / 2+\alpha / 2} \pi^{2} \Gamma\left(\frac{1}{2}+\alpha / 2\right)} \text { and } R^{2}=a^{2}+\rho^{2}+z^{2}-2 a \rho \cos t,
$$

or equivalently

$$
T^{*}=k_{1}(\alpha) e^{\lambda z} \int_{0}^{\pi} R^{-\alpha / 2-\frac{1}{2}} K_{-\alpha / 2-\frac{1}{2}}(\lambda R) \sin ^{\alpha} t d t,
$$

and

$$
T=k_{2}(\alpha) e^{\lambda z} \int_{0}^{\pi} R^{\alpha / 2-\frac{1}{2}} K_{\alpha / 2-\frac{1}{2}}(\lambda R) \sin ^{-\alpha} t d t,
$$

where

$$
k_{2}(\alpha)=\frac{M a^{-\alpha} \lambda^{\frac{1}{2}-\alpha / 2}}{2^{3 / 2-\alpha / 2} \pi^{2} \Gamma\left(\frac{1}{2}-\alpha / 2\right)} .
$$

For $r^{2}\left(=\rho^{2}+z^{2}\right)$ large it may be verified that the solutions (2.14) have the asymptotic behaviour of a point source of heat and that the solution (2.15) has the asymptotic behaviour of a point source of $\alpha$-heat. The choice of (2.14) or (2.15) as the unique solution of the original problem can only be made if the behaviour of $T$ or $T^{*}$ is specified on the $z$ axis in the statement of the problem. The most natural interpretation seems to be to regard (2.15) as the field of a ring source of heat and (2.14) as the field of a ring source of $\alpha$-heat. Then we impose $T^{*} \sim$ const. to get (2.14) or $T \sim$ a const. to get (2.15). This interpretation is possible because there is no qualitaive distinction to be made betewen a ring of heat and a ring of $\alpha$-heat. If $F(r)$ appears on the right hand side of the equation for $T$, then $\rho^{-\alpha} F(r)$ appears on the right hand side of the equation for $T^{*}$. But when $F(r)=(M / 2 \pi a) \rho^{-\alpha} \delta(\rho-a) \delta(z)$, because $f(x) \delta(x)=f(0) \delta(x)$ at a point of a continuity of $f$, the $\rho^{-\alpha}$ term becomes $a^{-\alpha}$. Thus a ring source of heat of total strength $M$ can also be regarded as a ring source of $\alpha$-heat, of total strength $M a^{-\alpha}$. It 
will therefore be not unexpected when we observe that if $a \rightarrow 0$ in equations (2.14) and (2.15) we obtain (2.7) if $a \rightarrow 0$, with $M$ fixed and (-2.8) (with $m=0$ ) if $a \rightarrow 0$ with $M a^{-\alpha}$ fixed.

\section{The solution of two boundary value problems}

In this section we will give some solutions of equation (1.1) which fit prescribed values of $T$ on the intervals $(-1,1)$, or $(0, \infty)$, and have a certain behaviour on the $z$ axis at points exterior to these intervals. The latter constraint is added in the light of the properties of the fundamental solutions constructed in the previous section.

We shall solve, in detail, the boundary value problem:

$$
\begin{gathered}
\frac{\partial^{2} T}{\partial z^{2}}+\frac{\partial^{2} T}{\partial \rho^{2}}+\frac{1-\alpha}{\rho} \frac{\partial T}{\partial \rho}-2 \lambda \frac{\partial T}{\partial z}=0, \\
T(z, 0)=f(z), \text { for }|z|<1, \\
T \rightarrow 0 \text { as } z^{2}+\rho^{2} \rightarrow \infty, \text { except possibly along } \rho=0, z>0,
\end{gathered}
$$

and

$$
T(z, \rho) \sim t_{1}(z) \text { as } \rho \rightarrow 0 \text { for }|z|>1 \text {. }
$$

Since $T \sim a$ constant, or $T \sim \rho^{\alpha}$, as $\rho \rightarrow 0$ for fixed $z$, the condition (3.4) specifies the former behaviour. The form of (3.4) emphasises that the value of the constant depends on $z$.

The problem will be solved using elliptic coordinates in an azimuthal plane. Set

$$
z+i \rho=\cosh (\xi+i \eta)
$$

then curves of constant $\xi$ are confocal ellipses with foci at $( \pm 1,0)$ and curves of constant $\eta$ are confocal hyperbolae with foci at $( \pm 1,0)$.

It is the equation for $e^{\lambda z} T$ which is separable thus with $V=e^{\lambda z} T$ we obtain

$$
\begin{aligned}
\frac{\partial^{2} V}{\partial \xi^{2}}+\frac{\partial^{2} V}{\partial \eta^{2}}+ & (1-\alpha) \operatorname{coth} \xi \frac{\partial V}{\partial \xi}+(1-\alpha) \cot \eta \frac{\partial V}{\partial \eta} \\
& -\lambda^{2}\left(\sinh ^{2} \xi+\sin ^{2} \eta\right) V=0
\end{aligned}
$$

so with $V=X(\xi) Y(\eta)$ we find

$$
X^{\prime \prime}+(1-\alpha) \operatorname{coth} \xi X^{\prime}-\left(k+\lambda^{2} \sinh ^{2} \xi\right) X=0,
$$

and

$$
Y^{\prime \prime}+(1-\alpha) \cot \eta Y^{\prime}-\left(-k+\lambda^{2} \sin ^{2} \eta\right) Y=0,
$$

where $k$ is the separation constant. 
The boundary condition is given on $\xi=0$ and so we are most concerned with choosing eigensolutions of the second equation (3.8). A complete mapping of the $z \rho$ plane is obtained for $\xi \geqq 0,0 \leqq \eta<\pi$ and it suffices to consider solutions of (3.8) which are even in $\eta$.

With $u=\cos \eta$ and $Y=\left(1-u^{2}\right)^{\alpha / 2} W$ equation (3.8) is replaced by

$$
\left(1-u^{2}\right) \frac{d^{2} W}{d u^{2}}-2 u \frac{d W}{d u}+\left(k+\frac{\alpha^{2}}{4}-\frac{\alpha^{2} / 4}{1-u^{2}}-\lambda^{2}\left(1-u^{2}\right)\right) W=0
$$

which is a particular form of the spheroidal differential equation. The book by Meixner and Schäfke (1954) contains an extensive study of this equation and Arscott (1964) and Erdélyi (1954; Volume III) also contain many useful properties. The substitution $\eta_{1}=\pi / 2-\eta$ transforms equation (3.8) to the Associated Mathieu equation which has been investigated thoroughly by Campbell (1955).

From the work of Meixner and Schäfke and of Campbell it can be proved that there exists an ennumerable sequence of values $k_{n}$ of $k$ such that the corresponding solutions $Y_{n}(\eta)$ of (3.8) are expressible as a series of Gegenbauer polynomials, thus

$$
Y_{n}(\eta)=\sum_{r=0}^{\infty} A_{n r}^{(\alpha)} C_{r}^{\left(\frac{1}{2}-\alpha / 2\right)}(\cos \eta)
$$

They are orthogonal on $(0, \pi)$ with weight function $(\sin \eta)^{1-\alpha}$, for $0<\alpha<2$, and

$$
\int_{0}^{\pi} Y_{n}(\eta) Y_{m}(\eta)(\sin \eta)^{1-\alpha} d \eta=\delta_{n m} \frac{n !}{\left(n+\frac{1}{2}-\alpha / 2\right) \Gamma(n+1-\alpha)}
$$

They are complete on $(0, \pi)$ spanning the space of functions which are analytic in a closed region of the complex plane which contains $(0, \pi)$ in its interior.

The transformations $u=\cosh \xi, X=\left(1-u^{2}\right)^{\alpha / 2} W$ applied to equation (3.7) give equation (3.9) again, but on this occasion our interests lie with solutions defined for $u>1$. For the values $k_{n}$ of $k$ which correspond to the solutions (3.10) the solutions of equation (3.9) defined for $u>1$ are expressible as series of Bessel functions. Any type of Bessel function is admissible but since it can be proved (Meixner and Schäfke, $\S 1.9$ ) that the series inherits the asymptotic form of the Bessel functions at infinity our choice is restricted to a series of modified Bessel functions; the solutions are thus

$$
X_{n}(\xi)=(\cosh \xi)^{\alpha / 2-\frac{1}{2}} \sum_{r=0}^{\infty} B_{n r}^{(\alpha)} K_{r+\frac{1}{2}-\alpha / 2}(\lambda \cosh \xi)
$$

From an examination of the behaviour of the solutions of equation (3.9) in the neighbourhood of the singular point $u=1$ and $u=-1$, it can be deduced that as $\xi \rightarrow 0$, 


$$
X_{n}(\xi) \sim \begin{cases}a \text { constant, } & \text { for } \alpha>0, \\ \log (\sinh \xi), & \text { for } \alpha=0, \\ (\sinh \xi)^{\alpha}, & \text { for } \alpha<0 .\end{cases}
$$

Since $\sinh \xi \sim \xi$ for $\xi$ small, then $\rho \sim \xi \sin \eta$ and $z \sim \cos \eta$, thus as $\rho \rightarrow 0$, for $|z|<1$ we have

$$
X_{n}(\xi) Y_{n}(\eta) \sim \begin{cases}f_{n}(z), & \text { for }>0, \\ f_{n}(z) \log \rho, & \text { for } \alpha=0, \\ f_{n}(z) \rho^{\alpha}, & \text { for } \alpha<0 .\end{cases}
$$

A similar argument also shows that when $\alpha>0, X_{n}(\xi) Y_{n}(\eta) \sim t_{n}(z)$ as $\rho \rightarrow 0$ for $|z|>1$. Thus it follows that the series

$$
T=e^{\lambda \cosh \xi \cos \eta} \sum_{n=0}^{\infty} a_{n} X_{n}(\xi) Y_{n}(\eta)
$$

is a solution of equations (3.1), (3.3) and (3.4). For $\alpha<0$ this expansion is inappropriate because of (3.14). However if we apply the result (1.14) to the series (3.15), we obtain a series for which $X_{n}(\xi) Y_{n}(\eta) \sim f_{n}(z)$ as $\rho \rightarrow 0$. But then the terms of this series satisfy $T^{*} \sim t_{n}(z)$ on $\rho=0,|z|>1$ and so the condition (3.4) will not be satisfied. The $a_{n}$ will be chosen to fit the boundary condition (3.2). For $\alpha<2$ they can be determined from the orthogonality relationships (3.11).

The asymptotic from of $T$ as given by (3.15) will follow the behaviour of $X_{n}(\xi)$ for a well behaved series, thus since

and

$$
X_{n}(\xi) \sim\left(e_{\xi}\right)^{\alpha / 2-1} \exp \left(\frac{-\lambda}{2} e^{\xi}\right), \text { as } \xi \rightarrow \infty,
$$

$$
r^{2}=\rho^{2}+z^{2} \simeq \frac{e^{2 \xi}}{4}, \eta \simeq \theta, \text { as } \xi \rightarrow \infty,
$$

then

$$
T \sim r^{\alpha / 2-1} \exp (-\lambda r(1+\cos \theta)) .
$$

This is the asymptotic form of the source of $\alpha$-heat and it enables us to regard the temperature field given by (3.15) as the field induced by a distributed source of $\alpha$-heat spread along the $z$ axis between $z=1$ and $z=-1$ whose density per unit length is adjusted so that it induces the prescribed temperature on the segment $\rho=0,|z|<1$.

The reader familiar with the properties of Mathieu functions will have noted the resemblance of the expansions (3.10) and (3.12) to the series for $c e_{n}(\eta)$ and $F_{e} k_{n}(\xi)$ respectively. The special case $\alpha=1$ gives these functions precisely the problem is then mathematically identical to the two dimensional problem for a heated plate set tangential to a uniform stream of inviscid heat conducting fluid (Belward (1969)). 
The case when the interval $(-1,1)$ is replaced by $(0, \infty)$ in the boundary value problem can be treated in an exactly similar manner. The problem retains all the main properties of the previous problem in respect to the dependence on $\alpha$. It is solved in terms of parabolic coordinates in the $z \rho$ plane. The mapping function is

$$
z+i \rho=(\xi=i \eta)^{2}
$$

The solution, for $\alpha>0$, is

$$
T=e^{-2 \eta^{2}} \sum_{n=0}^{\infty} a_{n} L_{n}^{(-\alpha / 2)}\left(2 \xi^{2}\right) \Psi\left(n+1-\frac{\alpha}{2}, 1-\frac{\alpha}{2} ; 2 \eta^{2}\right),
$$

where $L_{n}^{(a)}(w)$ is a Laguerre polynomial and $\Psi(a, b ; w)$ is a confluent hypergeometric function (see Erdélyi (1954); Volumes I and II)). The boundary conditions are applied on $\eta=0$ and the Laguerre polynomials are orthogonal and complete on $(0, \infty)$.

A solution which satisfies equations (3.2), (3.3), (3.1) and the condition $T^{*} \sim t_{n}(z)$ as $\rho \rightarrow 0$ can also be written down. One solution of interest is the special solution corresponding to the boundary condition $T=1$ on $\rho=0, z>0$. Then we have

$$
T=\Gamma^{-1}\left(\frac{\alpha}{2}\right) \Gamma\left(\frac{\alpha}{2}, 2 \eta^{2}\right),
$$

so that $T$ is constant on each paraboloid of revolution $\rho^{2}=4 \eta_{0}^{2}\left(z+\eta_{0}^{2}\right)$. The case of $\alpha=1$ which corresponds to the two dimensional problem is well known.

The parabolic wake appears explicity in these solutions. Along the curves $\eta=a$ constant the dependence is algebraic whereas across the curves the dependence is exponential.

\section{On integral representations and integral equations}

In the following paragraphs some integral representations of solutions of equation (1.1) will be given and the integral equation (1.2) will be constructed. It will be shown that the solution of the boundary value problems of the previous section can be used to solve equation (1.2). Finally we shall use two different integral representations of the same function to derive an inverse operator for equation (1.2) on $(-\infty, \infty)$.

The function

$$
\begin{aligned}
T(z, \rho)= & \frac{\lambda^{\frac{1}{2}-\alpha / 2} 2^{\alpha / 2-3 / 2}}{\pi^{3 / 2} \Gamma(1-\alpha / 2)} \int_{c}^{d} g(t) e^{\lambda(z-t)}\left[(z-t)^{2}+\rho^{2}\right]^{(\alpha-1) / 4} \\
& \cdot K_{(\alpha-1) / 2}\left(\lambda\left[(z-t)^{2}+\rho^{2}\right]^{\frac{1}{2}}\right) d t,
\end{aligned}
$$

is a solution of equation (1.1) provided only that $g$ is a generalised function of slow growth at infinity. When $g$ is integrable and $\alpha>0$, we have 


$$
\begin{aligned}
T(z, 0)=\frac{\lambda^{\frac{1}{2}-\alpha / 2} 2^{\alpha / 2-3 / 2}}{\pi^{3 / 2} \Gamma(1-\alpha / 2)} \int_{c}^{d} g(t) e^{\lambda(z-t)}|z-t|^{(\alpha-1) / 2} \\
\quad \times K_{(\alpha-1) / 2}(\lambda|z-t|) d t,
\end{aligned}
$$

which is essentially equation (1.2). Thus in the usual way the boundary value problem is reduced to an integral equation, and the solution of the integral equation gives the solution of the boundary value problem through the representation (4.1).

We can also show, more importantly, that the solution of the boundary value problem gives the solution of the integral equation. This follows by identifying the role of $g$ in the boundary value problem. We prove that

$$
g(z)=\lim _{\rho \rightarrow 0}\left(-2 \pi \rho^{1-\alpha} \frac{\partial T}{\partial \rho}\right), \text { for } \alpha<2 .
$$

First note that by comparison with the solution (2.8), with $m=0$, the field (4.1) is the field of a source of $\alpha$-heat distributed with strength $g(t)$ per unit length along the segment $(c, d)$. Thus $M^{*}$, the total flux of $\alpha$-heat from the segment $(c, d)$ is given by

$$
M^{*}=\int_{c}^{d} g(t) d t
$$

But $M^{*}$ can be calculated directly from $T$. If the segment $\rho=0, c<z<d$ is contained in the cylinder $0 \leqq \rho<a, h<z<k$ then the flux of $\alpha$-heat from the cylinder $M^{*}$ is

$$
M^{*}=\left.\int_{h}^{k}\left(-\frac{\partial T^{*}}{\partial \rho}-\frac{\alpha}{\rho} T^{*}\right) 2 \pi \rho\right|_{\rho=a} d z+\left.\int_{b}^{a}\left(-\frac{\partial T^{*}}{\partial z}+2 \lambda T^{*}\right)\right|_{z=h} ^{z=k} 2 \pi \rho d \rho .
$$

The cylinder can now be reduced in height and radius until it coincides with the segment $\rho=0, c<z<d$. We find that, in terms of $T$,

$$
M^{*}=\int_{c}^{d} \lim _{\rho \rightarrow 0}\left(-2 \pi \rho^{1-\alpha} \frac{\partial T}{\partial \rho}\right) d z, \text { for } \alpha<2,
$$

and (4.3) follows by comparison of (4.4) and (4.6). For $\alpha \geqq 2$ the integrals in (4.5) may not converge. A more rigorous proof of this result is given in another paper by Belward (to appear), where the integral equation (1.2) is given individual treatment.

The function

$$
\begin{array}{r}
T(z, \rho)=\frac{\lambda^{\frac{1}{2}+\alpha / 2} \rho^{\alpha}}{2^{3 / 2+\alpha / 2} \pi^{3 / 2} \Gamma(1-\alpha / 2)} \int_{c}^{d} h(t) e^{\lambda(z-t)}\left[(z-t)^{2}+\rho^{2}\right]^{-(\alpha-1) / 4} \\
\quad \times K_{-(\alpha-1) / 2}\left(\lambda\left[(z-t)^{2}+\rho^{2}\right]^{\frac{1}{2}}\right) d t .
\end{array}
$$


is also a solution of equation (1.1), and by comparison with (2.6) this is clearly the field of a distributed source of heat. From this we can deduce that

$$
h(z)=2 \pi \alpha T(z, 0) \text { for } c<z<d \text { and } \alpha>-2 .
$$

Note therefore that the function (4.7) is the solution of the boundary value problem in which (3.4) is replaced by the condition $T^{*} \sim t_{2}(z)$ for $z \notin(c, d)$, provided we put $h(z)=2 \pi \alpha f(z)$.

Finally note that when $T$ is given on the entire $z$ axis either representation is possible. This provides an elegant method of deducing an inverse operator for the integral equation (1.2). Simply replace $h(t)$ by $2 \pi \alpha f(t)$, then calculate $g(t)$ from (4.7) by computing

$$
\lim _{n \rightarrow 0}\left(-2 \pi \rho^{1-\alpha} \frac{\partial T}{\partial \rho}\right)
$$

The result is

$$
\begin{aligned}
g(t)=-\frac{\lambda^{\frac{1}{2}+\alpha / 2} \pi^{\frac{1}{2}} \alpha}{2^{\alpha / 2-3 / 2} \Gamma(\alpha / 2)} \int_{-\infty}^{\infty} f(z) e^{\lambda(t-z)}|t-z|^{-(\alpha-1) / 2} & \\
& \times K_{-(\alpha-1) / 2}(\lambda|t-z|) d z .
\end{aligned}
$$

The integral above does not converge in the ordinary sense when $0<\alpha<2$. However the statement of (4.9) as the inverse operator is valid for all $\alpha$ when interpreted as a relationship between distributions.

\section{Large and small Péclet numbers}

A feature of practical interest in these problems is the dependence of the solutions on the parameter $\lambda$, the Péclet number defined in section two. The boundary value problem in section three in which the temperature is specified on $(-1,1)$ is a problem in which the Péclet number is genuinely defined since there is a characteristic length in the problem. In this section some approximate solutions will be deduced for $\lambda$ large and for $\lambda$ small. The form of the solution (3.15) is extremely complicated and it seems impossible to deduce the dependence on $\lambda$ from it in terms of simple functions, so we use the integral equation formulation of the problem instead.

A second parameter of practical value in heat transfer problems is the Nusselt number. This is the nondimensioned heat flux into the fluid. In the problem of section three it is natural to generalise this notion and consider the total flux of $\alpha$-heat, $M^{*}$, into the fluid. The asymptotic dependence of $M^{*}$ on $\lambda$ can easily be established by using the approximate solutions of the integral equation. We impose the restriction $0<\alpha<2$, because $M^{*}$ is not in general finite for $\alpha \geqq 2$ and the kernel is not integrable for $\alpha \leqq 0$. 
The estimates are obtained by approximating the kernel of equation (1.2) so that the integral equation becomes a more simple equation whose solution can be given in closed form.

For large $\lambda$ we approximate the kernel of (1.2) by its asymptotic form. It is then exponentially small for positive values of its argument, when $\lambda$ is large, so we ignore this contribution to the integral. Equation (1.2) (for convenience assumed given on $(0,2)$ now) becomes the Abel equation

$$
\frac{\lambda^{-\alpha / 2} 2^{\alpha / 2-2}}{\pi \Gamma(1-\alpha / 2)} \int_{0}^{z} g(t)(z-t)^{\alpha / 2-1} d t=f(z) .
$$

The solution is

$$
g(z)=\lambda^{\alpha / 2} 2^{2-\alpha / 2} \pi \frac{d}{d z}\left(\frac{\Gamma(1-\alpha / 2)}{\Gamma(\alpha / 2) \Gamma(n+1-\alpha / 2)} \int_{0}^{z} f^{(n)}(w)(z-w)^{n-\alpha / 2} d w\right),
$$

where $n$ is chosen so that $-1<n-\alpha / 2-0$. Here it is assumed that $f^{(i)}(0)=0, i=0,1,2, \cdots, n-1$. While $M^{*}$ is not in general finite for $\alpha>2$ due to the non-integrability of $g$ at the origin, these conditions do ensure that $M^{*}$ is finite for large $\lambda$.

The dependence on $\lambda$, for $0<\alpha<2$, is demonstrated by the solution for $T(z, 0)=1$. In this case

$$
g(z)=\lambda^{\alpha / 2} \frac{\pi 2^{1-\alpha / 2} z^{-\alpha / 2}}{\Gamma(\alpha / 2)}
$$

and

$$
M^{*}=\lambda^{\alpha / 2} \frac{2^{4-\alpha} \pi}{(2-\alpha) \Gamma(\alpha / 2)} .
$$

The validity of these manipulations might be questioned because the approximation of the kernel is not uniform in $\lambda$. However the analysis is supported by two results. First a boundary layer analysis gives (5.3) precisely; and secondly if $g(z)$ (from (5.3)) is substituted into the full equation the integral can be shown to equal $1+O\left(\lambda^{-\alpha / 2}\right)$, thus the method is valid if 'small' right hand sides give 'small' solutions.

For $\lambda$ small and $0<\alpha<2$ the kernel can be approximated by

$$
w^{(\alpha-1) / 2} K_{(\alpha-1) / 2}(w) \simeq\left\{\begin{array}{l}
\Gamma\left(\frac{1-\alpha}{2}\right) 2^{-(\alpha+1) / 2} w^{\alpha-1}, 0<\alpha<1, \\
-\log \frac{1}{2} w \\
\frac{\Gamma\left(\frac{\alpha-1}{2}\right)}{2^{3 / 2-\alpha / 2}}+\frac{\Gamma\left(\frac{1-\alpha}{2}\right)}{2^{\frac{1}{2}+\alpha / 2}} w^{\alpha-1}, 1<\alpha<2,
\end{array}\right.
$$


and the three cases $0<\alpha<1, \alpha=1$, and $1<\alpha<2$ have to be considered separately. We outline the calculation for $0<\alpha<1$ and quote the remaining results. In all cases the dependence on $\lambda$ is obtained by taking $T(z, 0)=1$ on $0<z<2$. For $0<\alpha<1$.

$$
f(z) \simeq \frac{\Gamma\left(\frac{1}{2}-\alpha / 2\right)}{\Gamma(1-\alpha / 2)} \frac{1}{4 \pi^{3 / 2}} \int_{0}^{2} g(t)|z-t|^{\alpha-1} d t .
$$

Equation (5.6) is Carleman's equation, its solution is well known and is given, for instance, by Carrier et al. (1966). The solution is

$$
g(t)=\frac{4 \pi^{\frac{1}{2}}}{\Gamma\left(\frac{1}{2}-\alpha / 2\right) \Gamma(\alpha / 2)} t^{-\alpha / 2}(2-t)^{-\alpha / 2},
$$

and thus

$$
M^{*}=\frac{4 \pi \Gamma(1-\alpha / 2)}{\Gamma(\alpha / 2) \Gamma(1 / 2-\alpha / 2) \Gamma(3 / 2-\alpha / 2)} .
$$

Here we have the interesting result that $g$ and $M^{*}$ are independent of $\lambda$ to a first approximation.

For $\alpha=1$, the solution of the equation is

$$
g(z)=\left(1+\frac{M^{*} \log (\lambda / 2)}{2 \pi^{2}}\right) \frac{2}{\pi \log 2}\left(2 z-z^{2}\right)^{-\frac{1}{2}}
$$

and

$$
M^{*} \sim-2 \pi^{2}(\log \lambda)^{-1}
$$

For $1<\alpha<2$ we find

$$
g(z)=\left(1-k_{\alpha} \lambda^{1-\alpha} M^{*}\right) l_{\alpha} z^{-\alpha / 2}(2-z)^{-\alpha / 2},
$$

where

$$
k_{\alpha}=2^{\alpha-3} \Gamma\left(\frac{\alpha-1}{2}\right) \pi^{-3 / 2} \Gamma^{-1}\left(1-\frac{\alpha}{2}\right) \quad l_{\alpha}=\frac{\pi}{2 \Gamma(3 / 2-\alpha / 2) \Gamma(1+\alpha / 2)},
$$

and

$$
M^{*}=2^{3-\alpha} \pi^{3 / 2} \Gamma\left(1-\frac{\alpha}{2}\right) \Gamma\left(\frac{\alpha}{2}-\frac{1}{2}\right) \lambda^{\alpha-1}
$$

The approximations made in obtaining this last group of results are uniform over their range of application and hence there should be little doubt of their validity. The analysis could be made rigorous by writing the kernel of equation (1.2) as the sum of the leading term plus an error term. By applying the inverse operator for Carleman's equation to the resulting equation the magnitude of the error term could then be estimated. 


\section{Acknowledgement}

The contents of this paper are taken from a chapter of my Ph.D. Thesis. I would like to thank my supervisors, Professor A. F. Pillow and Dr. V. G. Hart, for their help and encouragement. I also wish to thank the governing bodies of the University of Queensland and the University of East Anglia for generous financial support towards a twelve month visit to the School of Mathematics and Physics, during which this paper was written.

\section{References}

F. M. Arscott (1964), Periodic Differential Equations (Pergamon Press, London, 1964).

J. A. Belward (1969), 'The solution of a Fredholm integral equation on a finite interval', Quart. Appl. Math. 27, 313-321.

J. A. Belward (to appear), 'On the relationship of some Fredholm integral equations of the first kind to a family of boundary value problem's, J. Math. Anal. and Applics.

J. C. Burns (1967), 'The iterated equation of generalised axially symmetric potential theory', J. Austral. Math. Soc. 7, 263-300.

R. Campbell (1955), Théorie générale de l'équation de Mathieu (Masson et cie, Paris, 1955).

G. F. Carrier, M. Krook, and C. E. Pearson (1966), Functions of a complex variable (McGrawHill, New York, 1966).

D. Colton (1969), 'Uniqueness theorems for axially symmetric partial differential equations', $J$. Math. Mech. 18, 921-930.

A. Erdélyi et al. (1954), Higher Trancendental Functions, Volumes I, II, III, (McGraw-Hill, New York, 1954).

B. G. Koroniev, 'On a plate lying on an elastic half-space whose modulus of elasticity is a power of the depth', Dokl. Akad. Nauk. SSR, 112.

J. Meixner and F. W. Schäfke (1954), Matheiusche Funktionen und Sphäroidfunktionen (SpringerVerlag, Berlin, 1954).

A. F. Pillow (1970), 'Diffusion and convection in viscous in viscous flow', Bull. Austral. Math. Soc. 2, 145-164.

A. Weinstein (1953), 'Generalised axially symmetric potential theory', Bull. Amer. Math. Soc. 59, 20-38.

University of Queensland

St. Lucia, Qld, Australia 